\title{
PLASMA DIAGNOSTICS WITH X-RAY LINES OF OXYGEN IN PUPPIS A
}

Kathryn F. Fischbach, Claude R. Canizares, Thomas H. Markert, Joan M. Coyne M.I.T., Cambridge, Mass., U.S.A.

Abstract: High resolution $X$-ray spectral observations of Puppis A were performed with the FPCS on Einstein. We use plasma diagnostics of 1 ines from OVII and OVIII to constrain the values of temperature, ionization timescale, and hydrogen column density.

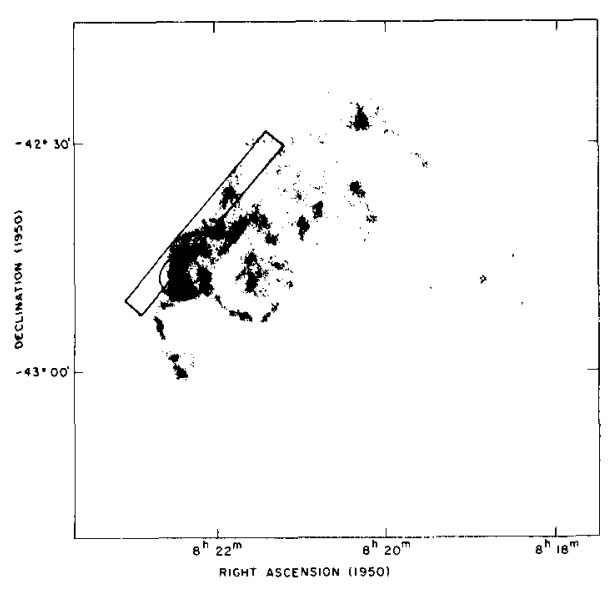

During November and December of 1979 , the shock front of the Puppis A supernova remnant was observed using the Focal Plane Crystal spectrometer (FPCS) on the Einstein observatory (Canizares et a1. 1979). A $3 \times 30$ arc minute aperture was aligned with the shock front, and RAP and TAP crystals were used to scan the energy ranges near the lines of hydrogen-like oxygen 10 VIII Lyman $\alpha, 630-670 \mathrm{eV}$ and 0 VIII Lyman $\beta, 750-800 \mathrm{eV}$ ) as well as the resonance, intercombination and forbidden lines $(n=2$ to $n=1$ transitions) of helium like oxygen (O VII $550-580 \mathrm{eV}$ ).

Figure 1

Figure 1 shows the FPCS aperture position for the observation of the shock front (rectangle) as well as the aperture position used for an observation of a bright knot (Winkler et al. 1983, circle) overlaid on the Puppis A image obtained by Petre et al. (1981) with the Einstein HRI. The raw FPCS spectra for the shock front appear in Figure 2, below. Note the differing background levels for the different observations.

The $X$-ray emission lines detected provide useful diagnostics of conditions in the shock front. For transitions $i \rightarrow g$ and $j \rightarrow k$ of ionlzation states $+x$ and $+y$ of an element $z$, the ratio of $X$-ray fluxes is given by: 


$$
\frac{f_{1 g}}{f_{j k}}=\frac{a_{1 g} \times n_{+x} \times \exp \left(-\sigma\left(E_{i g}\right) N_{H}\right) \times \exp \left(-E_{i g} /\left(k T_{e}\right)\right)}{n_{j k} \times n_{+y} \times \exp \left(-\sigma\left(E_{j k}\right) N_{H}\right) \times \exp \left(-E_{j k} /\left(k T_{e}\right)\right)}
$$

where $\Omega_{1 g}$ is the effective collision strength for transition $i \rightarrow g$ (see Vedder et al. 1986), Eig is the transition energy, $T_{e}$ the electron temperature, $n_{+x}$ the density of ion $x, \sigma$ the cross-section per hydrogen atom for photoelectric absorption at the energy $\mathrm{E}_{i g}$, and $\mathrm{N}_{H}$ the hydrogen column density. It is difficult to make very many precise measurements so as to be able to solve for all of the unknowns in the problem. The approach we have taken is to measure a few carefully chosen line intensities well so that as many parameters as possible will cancel.
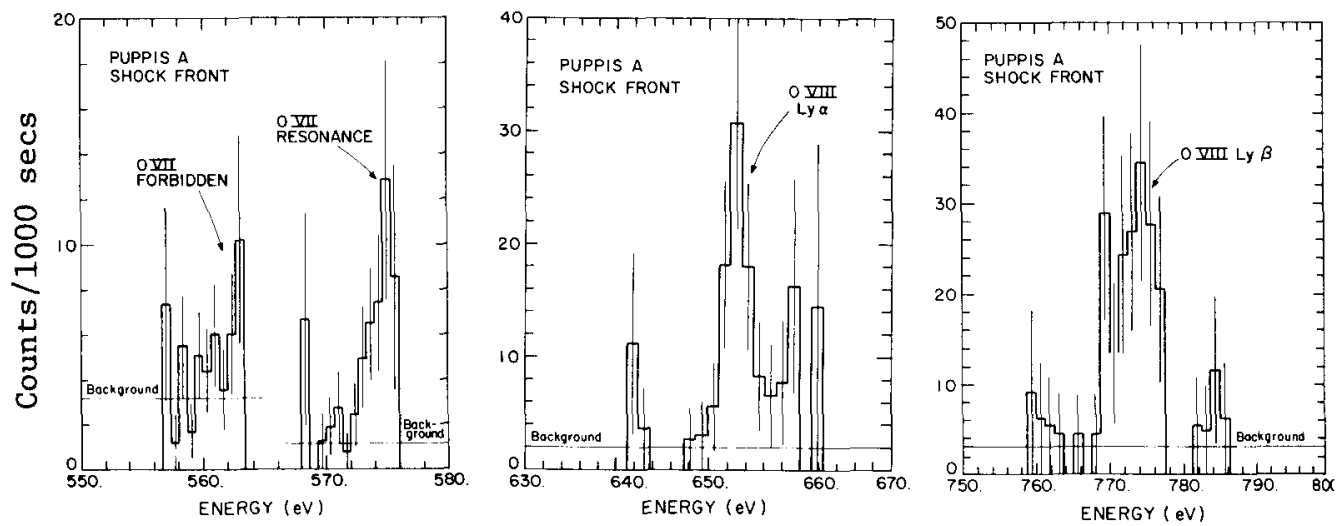

Figure 2 - FPCS Observations of Puppis A Shock Front

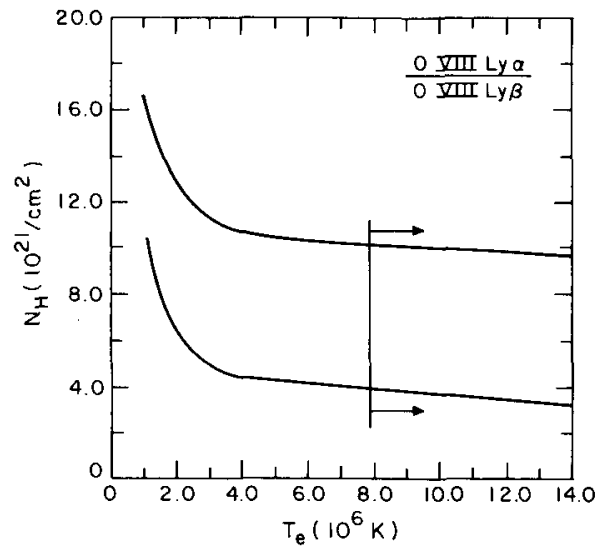

Figure 3

For example, a comparison of two shock front lines from the same ion, 0 VIII Ly $\alpha$ and 0 VIII Ly $\beta$, yields an allowed region in the parameter space of column density $\mathrm{N}_{\mathrm{H}}$ and electron temperature $\mathrm{T}_{\mathrm{e}}$ (see Figure 3). We have used collision strengths from shull (1981). We do not have a second ratio that serves to further confine the possible values of $\mathrm{N}_{\mathrm{H}}$, but, as shown below, we are able to exclude temperatures below $\approx 7.9 \times 10^{\circ}$ Kelvin, so that $\mathrm{N}_{\mathrm{H}}$ is restricted to a range of 3 to $10 \times 10^{21} \mathrm{~cm}^{-2}$.

The region allowed by the data is between the two curved lines and to the right of the vertical line. 
In order to use the other oxygen lines in Figure 2 to learn more about the shock front, it is necessary to perform a non-equilibrium analysis. This is required because the relative abundances of the varlous oxygen ions, which are functions of the time since the plasma was shocked, are parameters in the formulae for line fluxes. (For the analysis summarized in Pigure 3 a non-equilibrium approach is unnecessary since both lines are fron the same lon and collisional excitation will dominate in all plausible conditions.)

The ionization structure is determined by solving a set of $Z+1$ simultaneous differential equations. The flux ratios are then determined by using the resulting ion abundances explicitly in the equations for the line emissivities. We have used the technique of Hughes and Helfand (1985) to solve the lon balance equations. The complete analysis technique is discussed in more detail in Markert et al. (1987).

The line ratios of the oxygen lines constrain the various parameters of the non-equilibrium model. For the lines we have chosen these are $T_{e}, N_{H}$, and $\tau$. Here $\tau$ ( $\equiv$ electron density $\times$ time since the shock) is the ionization timescale and measures the extent to which ionization equilibrium has been attained.

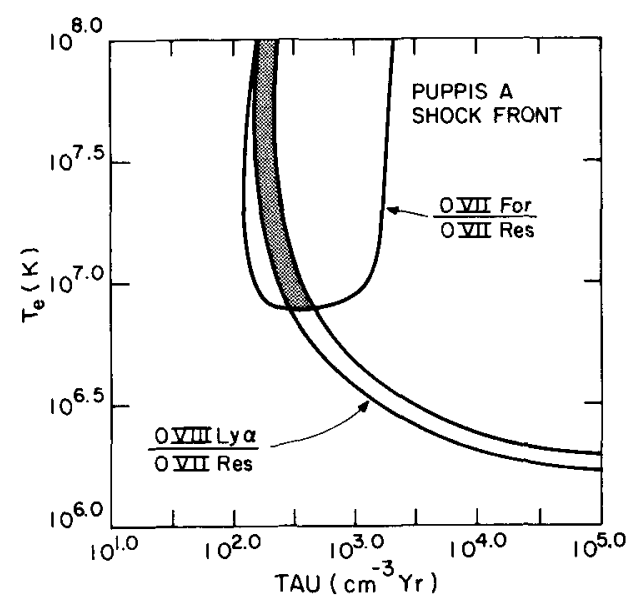

In Figure 4 we show $\pm 1 \sigma$ contours in $\left(\mathrm{T}_{\mathrm{e}}, \tau\right)$ parameter space which show the regions allowed by FPCS measurements of the line ratios 0 VIII Lyman $\alpha$ to 0 VII Resonance and 0 VII Forbidden to 0 VII Resonance. The overlapping (shaded) region is approximately the 90\% confidence region for the values of $T_{e}$ and $\tau$. For this analysis we have assumed $\mathrm{N}_{\mathrm{H}}=6 \times 10^{21} \mathrm{~cm}^{-2}$, consistent with Figure 3 , although in fact the results for $\left(T_{e}, \tau\right)$ are not particularly sensitive to the choice of $\mathrm{N}_{\mathrm{H}}$.

Pigure 4

In Table 1 (below) we list ranges of the parameters we have determined in the analyses shown in Figures 3 and 4 . We also include values for the parameters determined previously for two other regions of Puppis A: a bright knot of emission along the shock front (Winkler et al. 1983) and a region in the interior of the remnant (Winkler et al. 1981). 
Table 1

\begin{tabular}{lccc}
\hline & $N_{\mathrm{H}}\left(10^{21} \mathrm{~cm}^{-2}\right)$ & $\mathrm{T}_{\mathrm{e}}\left(10^{\circ} \mathrm{K}\right)$ & $\begin{array}{c}\mathrm{n}_{\mathrm{e}} \times \mathrm{t} \\
\left(\mathrm{yr} \mathrm{cm}^{-3}\right)\end{array}$ \\
\hline Shock Front & 3 to 10 & $\geqslant 7.9$ & 150 to 400 \\
$\frac{\text { Eastern }}{\text { Bright Knot }}{ }^{2}$ & $\approx 2$ & $\approx 7$ & $\approx 1000$ \\
Interior & 2 to 6 & $\geqslant 1.5$ & - \\
\hline
\end{tabular}

1 This paper

2 Winkler et al. 1983

3 winkler et al. 1981

Petre et al. 1981, have concluded that the eastern knot is a region where the shock front has encountered a cloud of density $\approx 10$ $\mathrm{cm}^{-3}$, implying (from the table) a time since shocking of 100 years. If the eastern knot and the shock front have the same age (a reasonable assumption because of the proximity of the two features), then we conclude that the density of the shock front is in the range 1.5 to 4.0 $\mathrm{cm}^{-3}$.

We thank Leah Bateman, Peter Vedder and Meg Urry for assisting In manuscript preparation, data analysis and for helpful discussions. We are indebted to Jack Hughes for supplying his computer code. This work was supported in part by NASA grant NAG 8-494.

\section{REFERENCES}

Canizares, C.R., Clark, G.W., Markert, T.H., Berg, C., Smedira, M., Bardas. D., Schnopper, H., and Kalata, K. 1979, Ap. J. (Letters), 234, L33.

Hughes, J.P. and Helfand, D.J. 1985, Ap. J., 291, 544.

Markert, T.H., Blizzard, P.L., Canizares, C.R. and Hughes, J.P. 1987, this conference.

Petre, R., Canizares, C.R., Kriss, G.A. and Winkler, P.F. 1981, Ap. J., 258,22 .

Shull, J.M. 1981, Ap. J. Suppl., 46, 27.

Vedder, P.W. Canizares, C.R., Markert, T.H. and Pradhan, A, 1986, Ap. J., $307,269$.

Winkler, P.F., Canizares, C.R., Clark, G.W., Markert, T.H., Petre, R. 1981, Ap. J., 245, 574 .

Winkler, P.F., Canizares, C.R., and Bromley, B.C. 1983, in Supernova Remnants and Their $X$-ray Emission, eds. $J$. Danziger and $P$. Gorenstein, (Dordrecht: Reidel), 245. 